\title{
Cosmological perturbations in the 5D big bang
}

\author{
Jaume Garriga \\ Departament de Física Fonamental, Universitat de Barcelona, Diagonal 647, 08028 Barcelona, Spain, \\ IFAE, Universitat Autònoma de Barcelona, 08193 Bellaterra, Barcelona, Spain, \\ and Yukawa Institute for Theoretical Physics, Kyoto University, Kyoto 606-8502, Japan \\ Takahiro Tanaka \\ Yukawa Institute for Theoretical Physics, Kyoto University, Kyoto 606-8502, Japan
}

(Received 25 December 2001; published 29 April 2002)

\begin{abstract}
Bucher has recently proposed an interesting brane-world cosmological scenario where the "big bang" hypersurface is the locus of collision of two vacuum bubbles which nucleate in a five-dimensional flat space. This gives rise to an open universe, where the curvature can be very small provided that $d / R_{0}$ is sufficiently large. Here, $d$ is the distance between bubbles and $R_{0}$ is their size at the time of nucleation. Quantum fluctuations develop on the bubbles as they expand towards each other, and these in turn imprint cosmological perturbations on the initial hypersurface. We present a simple formalism for calculating the spectrum of such perturbations and their subsequent evolution. We conclude that, unfortunately, the spectrum is very tilted, with a spectral index $n_{s}=3$. The amplitude of fluctuations at the horizon crossing is given by $\left\langle(\delta \rho / \rho)^{2}\right\rangle$ $\sim\left(R_{0} / d\right)^{2} S_{E}^{-1} k^{2}$, where $S_{E} \gg 1$ is the Euclidean action of the instanton describing the nucleation of a bubble and $k$ is the wave number in units of the curvature scale. The spectrum peaks on the smallest possible relevant scale, whose wave number is given by $k \sim d / R_{0}$. We comment on the possible extension of our formalism to more general situations where a big bang is ignited through the collision of $4 \mathrm{D}$ extended objects.
\end{abstract}

DOI: 10.1103/PhysRevD.65.103506

PACS number(s): $98.80 . \mathrm{Cq}$

\section{INTRODUCTION}

The possibility of having gravity localized [1,2] on a brane which moves in a higher dimensional space has recently stimulated the search for alternatives to the standard inflationary paradigm [3-5]. In these alternative scenarios, the big bang would result from the collision of 4-dimensional extended objects propagating in five dimensions. Pioneering work in this direction [4] did not use the localization of gravity as an essential ingredient, but this was at the expense of a somewhat singular behavior at the moment of collision [6], when the "bulk" in which the branes propagate momentarily disappears.

The generic predictions of inflation seem to be in good agreement with current cosmological data, and therefore any alternative proposal has to measure up to high standards. In particular, it is important to clarify the mechanisms by which cosmological perturbations are generated, since at the moment these provide the finest tests for any theory of initial conditions. The purpose of this paper is to consider this problem in the context of the model proposed in Ref. [3], where it seems to be best posed (see also [7]).

The paper is organized as follows. In Sec. II we briefly review Bucher's scenario, describing the geometry of the problem. In Sec. III we derive the expression for the primordial cosmological perturbations in terms of the physical parameters of the model. In Sec. IV we discuss the subsequent evolution of such perturbations, and in Sec. V we summarize our conclusions.

\section{A BIG BANG FROM BUBBLE COLLISION}

In Bucher's model one starts with a metastable (or "false") vacuum in five dimensions, which is flat space or a very mildly expanding de Sitter space. This decays through bubble nucleation into an anti-de Sitter (AdS) phase, where the five-dimensional cosmological constant is negative. The model requires the existence of degenerate discrete AdS vacua. Upon collision of two bubbles corresponding to different vacua, a domain wall forms, where gravity is localized in the manner of Randall and Sundrum [1]. The "big bang" hypersurface is the locus where the world sheets of the two bubbles meet, and the domain wall, or "local brane," is the place where we are supposed to live.

Let us use coordinates $X^{A}=\left(X^{i}, W, T\right)$ in the original 5D Minkowski space, where a pair of bubbles of radius $R_{0}$ nucleate at $T=0$, separated by a distance $2 d$. The radius $R_{0}$ is a fixed parameter of the theory, which is related to the bubble wall tension $\sigma$ and to the energy gap $\epsilon$ between the original Minkowski phase and the final AdS phases [8] (this relation, however, will not be needed in the following discussion.) Let us choose the origin of coordinates to be at the center of one of the bubbles. After nucleation, the bubble wall expands with constant acceleration, following a hyperbolic trajectory which can be parametrized as

$$
\begin{aligned}
& T=R_{0} \sinh \beta, \\
& R=R_{0} \cosh \beta,
\end{aligned}
$$

where $R \equiv\left(|\vec{X}|^{2}+W^{2}\right)^{1 / 2}$, and $\beta$ is the boost parameter of the bubble wall

$$
v=d R / d T=\tanh \beta
$$

The collision takes place on the plane $W=d$, along the hyperboloid 


$$
T^{2}-|\vec{X}|^{2}=d^{2}-R_{0}^{2} .
$$

To the future of this hyperboloid, and on the plane $W=d$, a domain wall forms where gravity is four dimensional $[1,9]$. Throughout this paper we shall assume that, locally, the collision process is almost instantaneous compared with the length scales of our interest, and that a fixed fraction of the energy of the collision is channeled into the degrees of freedom which live on the local brane. We shall also assume the usual fine tuning between the tension of the local brane and the AdS radius, so that the effective 4D cosmological constant is sufficiently small.

In the unperturbed setup, the 4D Minkowski space on the plane $W=d$ is matched into an open FRW model along the hypersurface (3). Along this hyperboloid, the metric is continuous, although its derivative is not (this jump in the extrinsic curvature corresponds to the jump in the Hubble rate accross the surface). It is convenient to use Milne coordinates to describe the 4D Minkowski space before the collision:

$$
{ }^{(4)} d s^{2}=-d t^{2}+t^{2} d \Omega_{H}^{2},
$$

where the $t=$ const surfaces have the hyperbolic geometry described by $d \Omega_{H}^{2}$. In terms of Minkowski coordinates, Milne time is given by $t=\left(T^{2}-|\vec{X}|^{2}\right)^{1 / 2}$. This form of the metric is valid for $t<R_{0} \cosh ^{-1}\left(d / R_{0}\right)$.

For $t>R_{0} \cosh ^{-1}\left(d / R_{0}\right)$, the 4D metric on the "local brane" is given by

$$
{ }^{(4)} d s^{2}=-d t^{2}+a^{2}(t) d \Omega_{H}^{2} \text {. }
$$

The local brane separates the interiors of the two vacuum bubbles, which have already collided and continue to expand. Due to the $O(3,1)$ symmetry of the process, the bulk metric on both sides of the local brane is given by Schwarzschild-AdS $[9,10]$. The evolution of the scale factor after collision is given by the usual Friedmann equation

$$
H^{2} \approx \frac{\kappa^{2}}{3} \rho+\frac{1}{a^{2}} .
$$

Here, $H=\dot{a} / a, \kappa^{2}=8 \pi G$ is the effective 4D gravitational coupling and $\rho$ is the matter energy density which is deposited on the local brane after collision. This will also include some "dark radiation" [9] because a significant fraction of the energy of the colliding bubbles may not stick to the brane; it may just fly into the bulk contributing to the effective mass of the Schwarzschild-AdS. In Eq. (5) we have also neglected "brany" corrections in the right-hand side, proportional to $\kappa^{4} \rho^{2} l^{2}$. These will be unimportant provided that the AdS radius $l$ in the bulk is sufficiently small. The discussion of cosmological perturbations with these corrections included is significantly more complicated and it is left for future research.

From Eq. (3), the spatial curvature scale of the open universe at the moment of collision is given by $\left(d^{2}-R_{0}^{2}\right)^{1 / 2}$, so the flatness problem is solved provided that $d$ is sufficiently large (as discussed in Sec. III A, this gives a lower bound on $\left.d / R_{0}\right)$. Of course, the homogeneity problem is also solved, due to the residual $O(3,1)$ symmetry of the colliding bubble setup along the hyperboloid (3). Thus, in principle, Bucher's scenario seems to provide an attractive starting point for a brane-world cosmology.

\section{INITIAL PERTURBATIONS}

Aside from solving the homogeneity and flatness problems, a serious candidate for a theory of initial conditions should explain the origin of the primordial cosmological perturbations. In brane collision scenarios these may be seeded by preexisting fluctuations in the shape of the colliding branes. These produce distortions of the "big bang" hypersurface as well as initial perturbations in the distribution of the energy density. In this section we estimate this effect for the case of colliding bubbles.

Quantum fluctuations on expanding vacuum bubbles have been studied in Refs. [11]. For a bubble which is centered at the origin of coordinates, the perturbed worldsheet $\widetilde{X}^{A}$ is conveniently parametrized as

$$
\tilde{X}^{A}\left(\xi^{\mu}\right)=X^{A}\left(\xi^{\mu}\right)+N^{A}\left(\xi^{\mu}\right) \chi\left(\xi^{\mu}\right)=\left(1+\chi / R_{0}\right) X^{A} .
$$

Here, $N^{A}$ is the unit normal to the unperturbed worldsheet of the bubble, which has the internal geometry of a 4D de Sitter space of radius $R_{0}, \xi^{\mu}$ are a set of coordinates in this space and $X^{A}$ stands for the unperturbed worldsheet (2). It is known [11] that the normal displacement $\chi$ behaves like a worldsheet scalar field with the tachyonic mass $m^{2}$ $=-4 R_{0}^{-2}$, which obeys an equation of the form ${ }^{1}$

$$
-\square \chi-4 R_{0}^{-2} \chi=0
$$

Here, $\square$ is the covariant d'Alembertian in a 4D de Sitter space.

We must also consider the second bubble, whose center is at a distance $2 d$ from the first. For perturbations which are symmetric with respect to the plane $W=d$, the collision will still take place on this plane, the second bubble being just a mirror image of the first. In what follows, we shall restrict our attention to such " $Z_{2}$ symmetric" perturbations. It is easy to show, following a calculation similar to the one presented below, that the antisymmetric mode has an amplitude comparable to that of the symmetric mode, but does not contribute to cosmological perturbations to linear order, so its effect seems to be much smaller than that of the symmetric mode.

An important observation is that, by conservation of momentum, the fluid lines will be orthogonal to the perturbed surface of collision, which is therefore a comoving, or ve-

\footnotetext{
${ }^{1}$ Here, and in the following discussion, we are neglecting the selfgravity effect of the bubble walls on the perturbations. Domain walls produce a repulsive constant gravitational force which causes an acceleration of order $(G \sigma)^{-1}$, where $G$ is the five-dimensional Newton's constant and $\sigma$ is the wall tension. If this length is very large compared with the inverse of the proper acceleration of the bubble wall, given by $R_{0}^{-1}$, then the self-gravity of the wall will be negligible.
} 
locity orthogonal surface [12]. It is known (see next section) that the growing mode of the curvature perturbation $\mathcal{R}_{c}$ on comoving surfaces is a constant of motion on scales larger than the Hubble rate, and hence $\mathcal{R}_{c}$ will be a quantity of interest to us. In the present case, however, it will be equally important to consider the decaying mode, since its initial amplitude is comparable to that of the growing mode. Therefore in order to characterize the initial perturbation we will also need the density perturbation $\delta_{c}$ on the same initial surface.

\section{A. Initial value of $\boldsymbol{R}_{c}$}

Due to local shifts $\delta t$ in the time of collision caused by fluctuations of the bubble shape, the big bang surface will no longer be the smooth hyperboloid (3). As explained in the preceeding paragraphs, we can restrict attention to perturbations which are symmetric with respect to the plane $W=d$. Then, to linear order in $\delta t$, the metric induced on the big bang surface is easily obtained from Eq. (4), and it is given by

$$
{ }^{(3)} d s^{2}=\left(1+2 \frac{\delta t}{t}\right) t^{2} d \Omega_{H}^{2} \text {. }
$$

Since we live in an almost flat universe, we shall be interested in length scales which are short compared to the curvature scale of the unperturbed surface. On these scales $d \Omega^{H}$ can be replaced with a flat metric. Then, the intrinsic curvature ${ }^{(3)} R$ of the perturbed surface is easily found to be

$$
{ }^{(3)} R=\frac{4}{t^{2}} \Delta \mathcal{R}_{c}=\frac{4}{t^{2}} \Delta \frac{\delta t}{t},
$$

where $\Delta$ is the comoving Laplacian. The first equality is just the conventional definition of the curvature perturbation used by most authors [16]. In a flat Friedmann-Robertson-Walker (FRW) with arbitrary scale factor and in an arbitrary gauge this definition takes the form

$$
{ }^{(3)} R \equiv \frac{4}{a^{2}} \Delta \mathcal{R},
$$

where now ${ }^{(3)} R$ is the perturbation of the intrinsic curvature scalar in the constant time surfaces of the corresponding gauge.

To proceed, we must find $\delta t$ as a function of the normal displacement $\chi$. Since all points on the unperturbed hyperboloid are equivalent, we may just consider the vicinity of the point $\vec{X}=0$, where $t=T$. It should be noted that the field $\chi$ lives on the unperturbed bubble, which hits the collision plane at some value $\beta_{d}$ of the unperturbed boost parameter determined by $d=R_{0} \cosh \beta_{d}$. However, the actual collision does not take place at that time, but at the time when the perturbed bubble reaches $W=d$, at some value $\beta_{c}$ of the unperturbed boost parameter given by $d=\left(R_{0}+\chi\right) \cosh \beta_{c}$. Thus, the shift in the time of collision is given by

$$
\begin{aligned}
\delta t=\delta T & =\widetilde{T}-T \\
& =\left(R_{0}+\chi\right) \sinh \beta_{c}-R_{0} \sinh \beta_{d} \\
& \approx-\frac{\chi}{\sinh \beta_{d}} .
\end{aligned}
$$

We conclude that the curvature perturbation on the initial comoving surface is given by

$$
\mathcal{R}_{c(i)}=\frac{\delta T}{T}=-\frac{\chi}{R_{0} \sinh ^{2} \beta_{d}} .
$$

Let us now estimate the size of this effect.

As mentioned above, $\chi$ satisfies the equation for a scalar field on the worldsheet de Sitter space, Eq. (6). The corresponding canonical field $\phi$ with dimensions of mass is related to $\chi$ by $[11]$

$$
\phi=\sigma^{1 / 2} \chi
$$

where $\sigma$ is the tension of the wall. As is well known, when a given mode of a nearly massless scalar field crosses the de Sitter horizon $R_{0}^{-1}$, it "freezes" with some amplitude $\phi_{k}$ $\sim R_{0}^{-1}$. However, in our case this amplitude does not stay constant. Due to the tachyonic mass $m^{2}=-4 H^{2}$ in Eq. (6), a mode with wave number $k$ grows proportionally to $e^{\beta}$ as

$$
\chi_{k}(\beta) \sim \sigma^{-1 / 2} R_{0}^{-1} e^{\beta-\beta_{k}},
$$

where $\beta_{k}$ is the value of the time-like boost parameter [see Eq. (2)] at which the physical wavelength of the mode $k^{-1} R_{0} e^{\beta}$ becomes larger than the inverse expansion rate on the bubble, $R_{0}$. Here we adopt the convention that the comoving curvature scale corresponds to $k \sim 1$. It is easy to see that at the time of collision we have $\exp \left(\beta_{d}-\beta_{k}\right)$ $\sim k^{-1}\left(d / R_{0}\right)$, and therefore,

$$
\chi_{k}\left(\beta_{c}\right) \sim \sigma^{-1 / 2} \frac{d}{R_{0}^{2}} k^{-1} .
$$

Using $\exp \beta_{d} \approx d / R_{0}$ in Eq. (8) we find that by order of magnitude,

$$
\mathcal{R}_{c(i)}(k) \sim\left(\frac{1}{S_{E}}\right)^{1 / 2} \frac{R_{0}}{d} k^{-1} .
$$

Here $S_{E} \sim \sigma R_{0}^{4} \gg 1$ is the Euclidean action of the instanton describing the nucleation of the bubble.

This perturbation is rather minute even on scales comparable to the curvature scale $k \sim 1$. An upper bound on $R_{0} / d$ can be obtained as follows. The spatial curvature radius of our present universe is much larger than the present Hubble radius $H_{0}^{-1}$. Taking into account that the curvature radius of the initial surface was given by $d$, this leads to the constraint

$$
z_{i} d \gtrsim H_{0}^{-1}
$$

where $z_{i}$ is the redshift at which the collision surface is. This redshift is given by 


$$
z_{i} \approx\left(\frac{\rho_{i}}{\rho_{0}}\right)^{1 / 4} \lesssim\left(\sigma \frac{d}{R_{0}} \frac{\kappa^{2}}{H_{0}^{2}}\right)^{1 / 4}
$$

where we assume radiation dominance for most of the cosmological evolution. In the last inequality we have used the fact that the energy density after collision will not be larger than the input energy density, which is of order $\sigma \cosh \beta_{d}$. From the two previous inequalities we find

$$
\frac{R_{0}}{d} \lesssim 10^{-24} S_{E}^{1 / 5}
$$

Therefore, we have

$$
\mathcal{R}_{c(i)}(k) \lesssim 10^{-24} S_{E}^{-3 / 10} k^{-1} .
$$

If there was no initial density perturbation, then this quantity would stay nearly constant until horizon reentry (see the next section). This would lead to an amplitude of perturbations smaller than the observed value by some 20 orders of magnitude. As we shall see, the bound (11) still applies to scales comparable to the curvature scale. However, on smaller scales the initial density perturbation $\delta_{c(i)}$ induces a much larger perturbation on $\mathcal{R}_{c}$ by the time of horizon reentry. Unfortunately, this comes at the prize of a very tilted spectrum, which is not compatible with observations.

\section{B. Initial value of $\delta_{c}$}

To evolve the cosmological perturbations, two initial conditions are needed for each wavelength. In addition to the initial curvature $\mathcal{R}_{c}$ we will also need the density perturbation $\delta_{c}$. By assumption, a fixed fraction of the energy of the collision goes into the brane, and therefore the density at the moment of collision is proportional to the Lorentz factor $\gamma$ $=\cosh \widetilde{\beta}$. Since the boost parameter is additive, the perturbed one will be given by

$$
\widetilde{\beta}=\beta_{c}+\dot{\chi}
$$

where

$$
\dot{\chi}=\frac{1}{R_{0}} \frac{\partial \chi}{\partial \beta}
$$

is the derivative of the perturbation with respect to the proper time $\tau=R_{0} \beta$ measured by an observer on the bubble. Thus, the change in the boost parameter is given to linear order by

$$
\delta \beta=\widetilde{\beta}-\beta_{d}=\dot{\chi}-\frac{\chi}{R_{0}} \operatorname{coth} \beta_{d}
$$

With these relations, we obtain the density perturbation at the moment of collision as

$$
\delta_{c(i)}=\tanh \beta_{d} \delta \beta=\dot{\chi} \tanh \beta_{d}-\frac{\chi}{R_{0}}
$$

As noted above, in the case of our interest, the rms fluctuation in $\chi$ grows exponentially fast with $\beta$. However, the combination which enters $\delta_{c}$ annihilates the leading term in this exponential dependence, and leaves only a contribution which decays with $\beta$. From this argument alone, it should be clear that the spectrum of $\delta_{c}$ will not be scale invariant, and that it will have opposite tilt to the spectrum of $\mathcal{R}_{c}$. To extract the spectral index, we need to look at the detailed form of the mode functions.

For definiteness, we shall use the open chart on the de Sitter worldsheet of the bubble. The conclusion, however, is independent of our slicing since we are interested only in wave-lengths much smaller than the curvature scale. In this chart, Eq. (6) for the evolution of $\chi$ reads

$$
\ddot{\chi}_{k}+\frac{3}{R_{0}} \operatorname{coth} \beta \dot{\chi}_{k}-\frac{4}{R_{0}^{2}} \chi_{k}+\frac{1+k^{2}}{R_{0}^{2} \sinh ^{2} \beta} \chi_{k}=0 .
$$

The quantum state of perturbations on a nucleating bubble is uniquely determined by de Sitter invariance [13]. It is given by the so-called Bunch-Davies vacuum (also known as the Euclidean vacuum). The corresponding modes in the open chart have been studied by $[14,15]$, and for a scalar field with mass $m^{2}=-4 R_{0}^{2}$ they are given by

$$
\chi_{k}(\beta) \propto \frac{P_{2}^{i k}(\cosh \beta)}{\sinh \beta},
$$

where $P_{2}^{i k}$ are the Legendre functions with the branch cut from -1 to 1 on the real axis. In the above equation we have ignored a contribution proportional to the decaying mode, which is accompanied by the factor $e^{-\pi k}$ and which is therefore irrelevant at wavelenths much shorter than the curvature scale. Expanding the Legendre function for large $\beta$, we have

$$
\chi_{k}(\beta) \sim \frac{\sigma^{-1 / 2} R_{0}^{-1}}{\sinh \beta_{k}}\left[\sinh \beta+\frac{k^{2}+4}{6 \sinh \beta}+O\left(e^{-3 \beta}\right)\right] .
$$

Here, the normalization is fixed as in Eq. (9), the only difference being that we have been careful to keep more terms in the expansion because the leading one clearly does not contribute to Eq. (12). Substituting Eq. (14) in Eq. (12) and using $\exp \beta \approx d / R_{0}$ we have

$$
\delta_{c(i)} \sim\left(\frac{1}{S_{E}}\right)^{1 / 2} \frac{R_{0}}{d} k
$$

For $k \sim 1$, this is of the same order as the curvature perturbation given in Eq. (10), where both are very small. However, Eq. (15) can be much larger on small scales. Unfortunately, this is due to a strong tilt in the power spectrum, corresponding to $n_{s}=3$ in the standard notation.

\section{EVOLUTION OF THE PERTURBATIONS}

Equations (8) and (12) provide the initial conditions (position and momentum, as it were) for the evolution of cosmological perturbations in the FRW phase. As mentioned above, since our universe is reasonably flat, the scales of 
interest to us will be much smaller than the curvature scale and it is a good approximation to consider perturbations to a flat FRW universe.

In an arbitrary gauge, the perturbed metric for scalar perturbations can be written as [16]

$$
\begin{aligned}
d \widetilde{s}^{2}= & a^{2}(\eta)^{2}\left\{-(1+2 A Y) d \eta^{2}-2 B Y_{i} d \eta d x^{i}\right. \\
& \left.+\left[(1+2 D Y) \delta_{i j}+2 E Y_{i j}\right] d x^{i} d x^{j}\right\}
\end{aligned}
$$

where $Y \propto e^{i \mathbf{k x}}$ is the appropriately normalized plane wave, and summation over modes with different wave number $\mathbf{k}$ is omitted. The vector and the traceless tensor constructed from $Y$ are defined by $Y_{i}=-k^{-1} Y_{, j}$, and $Y_{i j}=k^{-2} Y_{, i j}+\frac{1}{3} \delta_{i j} Y$. We also use convenient combinations of metric perturbations defined by

$$
\begin{gathered}
\mathcal{R}=D+\frac{1}{3} E, \\
k \sigma_{g}=E^{\prime}-k B,
\end{gathered}
$$

where a prime denotes differentiation with respect to $\eta$. $\mathcal{R}$ is the quantity related to the perturbation of the spatial scalar curvature of a time slice through Eq. (7), and $\sigma_{g}$ is related to the shear of the hypersurface normal vector field. ${ }^{2}$ The familiar gravitational potential in the Newton gauge is gauge invariantly defined as

$$
\Phi=-\mathcal{R}+\mathcal{H} k^{-1} \sigma_{g}
$$

where $\mathcal{H}=a^{\prime} / a$.

The energy momentum tensor of a perturbed perfect fluid is given by

$$
\widetilde{T}^{\mu \nu}=(\tilde{\rho}+\widetilde{P}) \tilde{u}^{\mu} \tilde{u}^{\nu}+\widetilde{P} \tilde{g}^{\mu \nu},
$$

with $\tilde{\rho}=\rho+Y \delta \rho, \widetilde{P}=P+Y \delta P, \tilde{u}^{0}=a^{-1}(1-A Y)$, and $\tilde{u}^{i}$ $=a^{-1} v Y^{i}$. We shall assume that the fluid consists of a single component. Then the ratio between the density and pressure perturbations becomes a function of the background energy density. We denote this ratio by $c_{s}^{2}=\delta P / \delta \rho$.

In the comoving gauge, in which $v-B=0$, the perturbed Einstein equations become

$$
\mathcal{H} A=\mathcal{R}_{c}^{\prime},
$$

and

$$
\mathcal{H}\left(k \sigma_{g}\right)-k^{2} \mathcal{R}_{c}=-\frac{\kappa^{2} a^{2}}{2} \delta \rho,
$$

\footnotetext{
${ }^{2}$ For the present discussion, the geometric interpretation of $\sigma_{g}$ will be irrelevant, it will just be used as a convenient variable.
}

$$
\begin{aligned}
\left(k \sigma_{g}\right)^{\prime}+2 \mathcal{H}\left(k \sigma_{g}\right)-k^{2}\left(\frac{\mathcal{R}_{c}^{\prime}}{\mathcal{H}}+\mathcal{R}_{c}\right) & =0, \\
\frac{\mathcal{H}^{\prime}-\mathcal{H}^{2}}{\mathcal{H}} \mathcal{R}_{c}^{\prime} & =\frac{\kappa^{2} a^{2}}{2} \delta P .
\end{aligned}
$$

Equations (20), (21) and (22) have already been simplified by using Eq. (19). For reference, we also quote the background equations

$$
\mathcal{H}^{\prime}=-\frac{\kappa^{2} a^{2}}{6}(\rho+3 P), \quad \mathcal{H}^{2}=\frac{\kappa^{2} a^{2}}{3} \rho
$$

Equations (19) and (20) give the following expression for the gauge invariant $\Phi$ in terms of the density perturbation $\delta_{c}$ :

$$
\Phi=-\frac{3 \mathcal{H}^{2}}{2 k^{2}} \delta_{c}
$$

The value of $\Phi$ is larger than $\delta_{c}$ by the factor of $\mathcal{H}^{2} / k^{2}$, and hence this is not a particularly illuminating variable on very large scales.

The evolution equations for given initial values for $\mathcal{R}_{c}$ and $\delta_{c}$ can be obtained by combining Eqs. (20), (21) and (22):

$$
\frac{d \mathcal{R}_{c}}{d N}=-c_{s}^{2} \frac{\delta_{c}}{(1+w)}
$$

$$
\frac{\mathcal{H}}{a^{2}} \frac{d}{d N}\left(a^{2} \mathcal{H} \delta_{c}\right)=k^{2}(1+w) \mathcal{R}_{c},
$$

where $w=P / \rho$ is the parameter characterizing the equation of state and $d N=\mathcal{H} d \eta$.

To discuss the evolution of perturbations it is useful to formally integrate the second equation,

$$
\delta_{c}=\frac{1}{a^{2} \mathcal{H}} \int_{N_{i}}^{N}\left(a^{2} \mathcal{H}\right) \frac{k^{2}}{\mathcal{H}^{2}}(1+w) \mathcal{R}_{c} d N+\frac{1}{a^{2} \mathcal{H}}\left(a^{2} \mathcal{H} \delta_{c}\right)_{(i)}
$$

The function $a^{2} / \mathcal{H}$ is an increasing function, so provided that $\mathcal{R}_{c}$ stays approximately constant at late times (which will be a self-consistent assumption), the integral in the right-hand side is dominated by the contribution from the neighborhood of the upper boundary of integration. If we consider a simple case in which $c_{s}^{2}=w=$ const, the scale factor and $\mathcal{H}$ are given by

$$
a=\left(\eta / \eta_{0}\right)^{2 /(1+3 w)}, \quad \mathcal{H}=\frac{2}{1+3 w} \eta^{-1}
$$

Performing the integral in Eq. (27) we have 


$$
\delta_{c} \approx \frac{2+2 w}{5+3 w} \frac{k^{2}}{\mathcal{H}^{2}} \mathcal{R}_{c}+\frac{1}{a^{2} \mathcal{H}}\left(a^{2} \mathcal{H} \delta_{c}\right)_{(i)},
$$

where we have assumed $\mathcal{R}_{c} \approx$ const at late times. ${ }^{3}$ Initially, $\mathcal{R}_{c} \lesssim \delta_{c}$ and consequently the first term in the right-hand side is suppressed with respect to the second at least by a factor of $\left(k^{2} / \mathcal{H}^{2}\right) \ll 1$. Substituting the dominant part into Eq. (25), we have

$$
\mathcal{R}_{c(f)} \approx \mathcal{R}_{c(i)}-\left(a^{2} \mathcal{H} \delta\right)_{(i)} \int_{\eta_{i}}^{\infty} \frac{d \eta}{a^{2}} \frac{c_{s}^{2}}{1+w}
$$

Then, this integration is performed to obtain

$$
\mathcal{R}_{c(f)} \approx \mathcal{R}_{c(i)}-\frac{2 w}{3\left(1-w^{2}\right)} \delta_{c(i)} .
$$

In obtaining Eq. (31) we have neglected the contribution from the first term in the right-hand side of Eq. (29). It is easy to check that this term does not have any effect until the wavelength of the mode is comparable to the Hubble radius $k \sim \mathcal{H}$. At that time, the second term in Eq. (29) is unimportant, and the amplitude of density perturbations at horizon crossing can be read off from the first term

$$
\left.\delta_{c}\right|_{h c} \approx \frac{2+2 w}{5+3 w} \frac{k^{2}}{\mathcal{H}^{2}} \mathcal{R}_{c(f)} .
$$

Finally, the Newtonian potential at horizon crossing can be found from the relation (24)

$$
\left.\Phi\right|_{h c} \approx-\frac{3+3 w}{5+3 w} \mathcal{R}_{c(f)}
$$

Substituting Eq. (31) into the previous two equations and using Eqs. (10) and (15) we have

$$
\left.\left.\Phi\right|_{h c} \sim \delta_{c \mid}\right|_{h c} \sim \mathcal{R}_{c(f)} \sim \frac{R_{0}}{d} S_{E}^{-1 / 2} k,
$$

which of course displays the same strong spectral tilt as the initial density perturbation $\delta_{c(i)}$.

\section{CONCLUSIONS}

In this paper we have discussed the primordial spectrum of density perturbations in the brane-world model proposed by Bucher [3]. In this scenario, two bubbles nucleate in the 5-dimensional Minkowski bulk, and their collision forms a brane where gravity is localized. This model solves the homogeneity and flatness problems, provided that the separation $2 d$ of the bubble nucleation points is sufficiently large

\footnotetext{
${ }^{3}$ Incidentally, even when $\mathcal{R}_{c}$ is not constant, but increasing with time, the order of magnitude of the first term in Eq. (29) does not change.
}

compared with the bubble radius $R_{0}$.

We have evaluated the initial spectrum of scalar density perturbations, assuming that they originate from quantum fluctuations on the bubbles. An important observation is that the surface of collision coincides with the so-called comoving hypersurface (or velocity orthogonal slicing). On this initial surface, we first evaluated the density contrast $\delta_{c}$ $=\delta \rho / \rho$ and the spatial curvature perturbation $\mathcal{R}_{c}$ [see Eq. (16) for definition]. The initial value $\delta_{c(i)}$ turns out to have a very steep spectrum corresponding to $n_{s}=3$, while the spectral index for $\mathcal{R}_{c(i)}$ is $n_{s}=-1$. The amplitude of both perturbations is comparable at wavelengths of the order of the curvature scale, and therefore $\delta_{c(i)}$ has a larger amplitude on scales relevant to present observations.

It is known that the curvature perturbation in the comoving gauge, $\mathcal{R}_{c}$, is conserved on scales much larger than the horizon, and therefore this quantity is often used to discuss the evolution of perturbations in the early universe. The constancy of $\mathcal{R}_{c}$, however, does not hold for the decaying mode. In the present case, the initial conditions which arise as a result of bubble collision contain a significant amount of "contamination" from the decaying mode. Therefore, $\mathcal{R}_{c}$ does not stay constant in the subsequent evolution of perturbations, which is described by two coupled first order differential equations. Given initial values $\delta_{c(i)}$ and $\mathcal{R}_{c(i)}$, we solved these equations to find that the final value of $\mathcal{R}_{c}$ (at the time of horizon crossing) becomes comparable to the initial value of the density perturbation $\delta_{c(i)}$ [see Eq. (30)]. This final value of $\mathcal{R}_{c}$ also gives the order of magnitude of the density contrast at horizon crossing. Thus, we conclude that the spectral index for primordial density perturbations is $n_{s}=3$. The estimated amplitude is very small at wavelengths comparable to the curvature scale. Perturbations of $O\left(10^{-5}\right)$ at the present horizon scale may of course be obtained by choosing the ratio between the curvature scale and the present horizon scale appropriately, but the spectrum is too steep to be consistent with observations.

Although in this paper we have investigated a particular realization of the brane big bang, we may have learned a few lessons which may be useful in more general cases. First, in the present model the bubble fluctuations are described by an effective 4-dimensional scalar field with a negative mass squared. Consequently, fluctuations on the bubble worldsheet have a red spectrum, which has a larger amplitude for longer wavelengths. However, the resulting spectrum of density perturbations turned out to be a blue one. This means that in principle it may be possible (although perhaps not easy) to generate a nearly scale invariant spectrum even when the mass of the effective field corresponding to the bubble fluctuations is not close to zero. Second, we need to be careful in using "standard" results of cosmological perturbation theory, which in some cases neglect the contribution from the decaying mode. In order to determine the evolution just after collision, two initial conditions must be supplied, and both turn out to be important.

Note added in proof. This paper was largely motivated by the work of M. Bucher and J.J. Blanco-Pillado [7]. We thank these authors for enlightening discussions and for en- 
couraging us to publish our results independently. In particular J.G. is grateful to M. Bucher for early discussions on the problem of cosmological perturbations generated in bubble collisions, and for sharing an early version of his manuscript during the Cambridge workshop on M-theory Cosmology, which eventually led to Ref. [7]. After our paper was complete a revised and extended version of [7] appeared where the collision of bubbles which nucleate in a de Sitter background is also considered.

\section{ACKNOWLEDGMENTS}

We are grateful to Jose Juan Blanco-Pillado and Martin Bucher for very useful discussions. J.G. is partially supported by the Templeton Foundation under grant COS 253, by CICYT under grant AEN98-0431 and by the Yamada Foundation. T.T. is partially supported by the Monbukagakusho Grant-in-Aid No. 1270154, and by the Yamada Foundation.
[1] L. Randall and R. Sundrum, Phys. Rev. Lett. 83, 3370 (1999); 83, 4690 (1999).

[2] G. Dvali, G. Gabadadze, and M. Porrati, Phys. Lett. B 485, 208 (2000).

[3] M. Bucher, hep-th/0107148.

[4] J. Khoury, B.A. Ovrut, P.J. Steinhardt, and N. Turok, Phys. Rev. D 64, 123522 (2001); R. Kallosh, L. Kofman, and A. Linde, ibid. 64, 123523 (2001); J. Khoury, B.A. Ovrut, N. Seiberg, P.J. Steinhardt, and N. Turok, ibid. 65, 086007 (2002).

[5] U. Gen, A. Ishibashi, and T. Tanaka, hep-th/0110286.

[6] Consequently, the determination of the spectrum of cosmological perturbations in this model seems to be a somewhat challenging problem. See, e.g., J. Khoury, B.A. Ovrut, P.J. Steinhardt, and N. Turok, hep-th/0109050; J. Hwang, Phys. Rev. D 65, 063514 (2002); S. Tsujikawa, Phys. Lett. B 526, 176 (2002); D.H. Lyth, ibid. 526, 173 (2002).

[7] This issue has been independently discussed by J. BlancoPillado and M. Bucher, Phys. Rev. D 65, 083517 (2002).

[8] S. Coleman and F. De Luccia, Phys. Rev. D 21, 3305 (1980).

[9] T. Shiromizu, K. Maeda, and M. Sasaki, Phys. Rev. D 62, 024012 (2000); P. Binetruy, C. Deffayet, U. Ellwanger, and D. Langlois, Phys. Lett. B 477, 285 (2000); E.E. Flanagan, S.H.
Tye, and I. Wasserman, Phys. Rev. D 62, 024011 (2000); J. Garriga and T. Tanaka, Phys. Rev. Lett. 84, 2778 (2000); J. Garriga and M. Sasaki, Phys. Rev. D 62, 043523 (2000).

[10] P. Bowcock, C. Charmousis, and R. Gregory, Class. Quantum Grav. 17, 4745 (2000).

[11] J. Garriga and A. Vilenkin, Phys. Rev. D 45, 3469 (1992); J. Garriga, ibid. 49, 6327 (1994).

[12] D. Langlois, K. Maeda, and D. Wands, Phys. Rev. Lett. (to be published), gr-qc/0111013.

[13] B. Allen, Phys. Rev. D 32, 3136 (1985); T. Vachaspati and A. Vilenkin, ibid. 43, 3846 (1991).

[14] M. Sasaki, T. Tanaka, and K. Yamamoto, Phys. Rev. D 51, 2979 (1995); K. Yamamoto, M. Sasaki, and T. Tanaka, Astrophys. J. 455, 412 (1995).

[15] M. Bucher, A.S. Goldhaber, and N. Turok, Nucl. Phys. B43, 173 (1995).

[16] For review of the standard cosmological perturbation see, e.g., A.R. Liddle and D.H. Lyth, Cosmological Inflation and Largescale Structure (Cambridge University Press, Cambridge, England, 2000); H. Kodama and M. Sasaki, Prog. Theor. Phys. Suppl. 78, 1 (1984); V.F. Mukhanov, H.A. Feldmann, and R.H. Brandenberger, Phys. Rep. 115, 203 (1992). 